\title{
TCF7L2 polymorphisms, nut consumption, and the risk of metabolic syndrome: a prospective population based study
}

\author{
Somayeh Hosseinpour-Niazi', Bahar Bakhshi', Asiyeh-Sadat Zahedi², Mahdi Akbarzadeh², \\ Maryam S. Daneshpour $2^{2_{+}}$(D), Parvin Mirmiran ${ }^{3^{*}+}$ (10 and Fereidoun Azizi ${ }^{4}$
}

\begin{abstract}
Background: The aim of this study was to investigate whether two variants of the TCF7L2 (rs7903146 and rs 12255372) modify the association between nut consumption and the risk of metabolic syndrome (MetS). Additionally, the modifying effect of weight change during follow-up on these associations was investigated.

Material and methods: We prospectively studied 1423 participants of the Tehran Lipid and Glucose study aged 19-74 years who were followed-up for dietary assessment using a validated, semi-quantitative food frequency questionnaire. Multivariable-adjusted Cox regression was used to estimate hazard ratios (HRs) for MetS events. Genotyping was performed by Human Omni Express-24-v1-0 chip.

Results: Over a median 8.9 years of follow-up, 415 new cases of MetS were documented. The median nut consumption was $20.0 \mathrm{~g} /$ week (Interquartile Range (IQR): 8.6-38.9 g/week). Regarding the rs 7903146 genotype, in carriers of T allele $(C T+T T)$, highest tertile of nut consumption was associated with a reduced risk of MetS after adjusting for confounders (HR: 0.67 (0.50-0.91)). Regarding the rs12255372 genotype, highest versus lowest tertile of nut consumption in participants with T allele (GT+TT) resulted in 34\% reduction of MetS risk after adjustment for confounders (HR: 0.66 (0.49-0.69)). After stratification by weigh change ( $<7 \%$ or $\geq 7 \%$ weight gain), in individuals with $\geq 7 \%$ weight gain, highest tertile of nut consumption was associated with reduced risk of MetS among the risk allele of rs7903146. In the risk allele of rs 12255372, among individuals with $<7 \%$ weight gain, third tertile of nuts intake reduced the risk of MetS, after adjustment for confounders.
\end{abstract}

Conclusion: Higher consumption of nuts may reduces the risk of MetS in T-risk allele of the TCF7L2 rs7903146 and rs12255372 variants and weight change may modify this association.

Keywords: Nuts, Metabolic syndrome, TCF7L2 polymorphisms, Gene-diet interaction, Weight change

*Correspondence: daneshpour1388@gmail.com; mirmiran@endocrine.ac.ir ${ }^{\dagger}$ Maryam S. Daneshpour and Parvin Mirmiran have contributed equally to this work and were equal in status

${ }^{2}$ Cellular and Molecular Research Center, Research Institute for Endocrine Sciences, Shahid Beheshti University of Medical Sciences, No 24, A'rabi St, Yeman Av, P.O. Box 19395-4763, Velenjak, Tehran, Iran

${ }^{3}$ Department of Clinical Nutrition and Dietetics, Faculty of Nutrition Sciences and Food Technology, National Nutrition and Food Technology Research Institute, Shahid Beheshti University of Medical Sciences, No 24, A'rabi St, Yeman Av, P.O. Box 19395-4763, Velenjak, Tehran, Iran

Full list of author information is available at the end of the article

\section{Introduction}

Metabolic syndrome (MetS) is a multicomponent condition characterized by insulin resistance, dyslipidemia, abdominal obesity, and hypertension that has well-documented associations with an increased risk of type 2 diabetes mellitus, cardiovascular disease (CVD), and atherosclerosis $[1,2]$. MetS has a multifactorial etiology, involving both genetics and modifiable environmental factors, including dietary determinants [3]. Identifying potential genes that are associated with MetS original author(s) and the source, provide a link to the Creative Commons licence, and indicate if changes were made. The images or other third party material in this article are included in the article's Creative Commons licence, unless indicated otherwise in a credit line to the material. If material is not included in the article's Creative Commons licence and your intended use is not permitted by statutory regulation or exceeds the permitted use, you will need to obtain permission directly from the copyright holder. To view a copy of this licence, visit http://creativecommons.org/licenses/by/4.0/. The Creative Commons Public Domain Dedication waiver (http://creativeco mmons.org/publicdomain/zero/1.0/) applies to the data made available in this article, unless otherwise stated in a credit line to the data. 
and investigating the modifying effect of genetics on the association of dietary determinants with the risk of MetS are among novel approaches for preventing and treating MetS. This approach is based on the precisionbased healthcare that enables use of therapeutic diets and dietary recommendations according to patients' genetic background that eventually, will lead to better prevention and treatment of MetS [4].

Among different single nucleotide polymorphisms (SNPs), the transcription factor-7-like 2 (TCF7L2) rs7903146 and rs12255372 are assumed to be associated with type 2 diabetes as well as MetS in different populations $[5,6]$. For the first time, the Diabetes Prevention Program and the Diabetes Prevention Study reported that lifestyle intervention reduces diabetic risk among individuals with genetic susceptibility of TCF7L2 risk genotypes $[7,8]$. Followed by this investigation, current literature suggests associations of this gene with the risk of type 2 diabetes, obesity, and MetS while considering the modulating effects of some dietary determinants, such as whole-grains [9], glycemic index and glycemic load [10], dietary fiber [11], low fat and high carbohydrate diet [12], dietary saturated fatty acids (SFA) [13], western dietary pattern [14], and prudent dietary pattern [15]. However, to the best of our knowledge, the interaction between nut consumption and polymorphisms of this gene has not yet been investigated. Nuts, as rich sources of fiber, polyphenols, mono- and polyunsaturated fatty acids, vitamins, minerals, and protein [16], could potentially reduce the risk of chronic diseases [17-21]. Although the inverse association between nut consumption and BMI, overweight, and obesity are documented in previous research [22, 23], limited studies have assessed the interaction between obesity, nut consumption, and chronic disease [24, 25]. Moreover, recently, an interaction between TCF7L2 and obesity in relation to type 2 diabetes has been reported [26, 27]. Therefore, the aim of the current study was to investigate (1) the association of nut consumption with the MetS risk based on the TCF7L2 rs7903146 and rs12255372 genotypes, and (2) whether these associations are modified according to weight change during follow-up ( $\geq$ or $<7 \%$ ) among adult participants of this population-based study, over a median 8.9-year of follow-up.

\section{Materials and methods Study population}

We conducted a prospective population-based study within the framework of the Tehran Lipid and Glucose Study (TLGS). The TLGS is an ongoing prospective study, aiming to prevent non-communicable diseases through promoting a healthy lifestyle. The detailed
TLGS method, described elsewhere [28], was initiated in March 1999. Multistage, stratified cluster random sampling technique was used for enrollment of $>15,000$ individuals aged $\geq 3$ years from Tehran's urban district 13 , a district with a population representative of the urban population of Tehran, the capital city of Iran. Beginning in 1999 with 3-year intervals afterwards, participants were assessed for demographic and lifestyle factors, socioeconomic status, medication use, medical history of cardiovascular risk factors, and anthropometric measures. To update all the previous data, the information were documented every 3 years during face-to-face visits by the local research team. Phases II, III, IV, V, VI were prospective follow-up studies conducted between 2002-2004, 2006-2007, 2008-2011, 2012-2015, 2016-2018, respectively. The current study used the baseline examination data from phase III of the TLGS (2006-2007) and followed participants up to phase VI of TLGS (2016-2018), during an 8.91-year follow-up (Interquartile range (IQR): 7.98-9.69).

During the third examination survey of the TLGS (2006-2007), a number of 12,523 participants were assessed for medical history and physical examinations. Subsequently, a representative sample of 4920 participants, based on their age and gender, was randomly selected to complete the dietary assessment. From the 4920 selected participants in the present study, 3462 number of individuals, with similar characteristics to the total population in phase III of the TLGS, agreed to complete the food frequency questionnaire (FFQ) [29]. For the current study, of 3462 participants, 3265 adults aged 19-74 years with complete demographic, anthropometric, biochemical, and dietary data were selected. Moreover, participants with MetS at baseline $(n=879)$, participants who were pregnant or lactating at baseline and during follow-up $(\mathrm{n}=28)$, participants with daily energy intake $<500$ and $>4200 \mathrm{kal}$ per day $(n=115)$, participants following any specific diet $(n=26)$, and participants with missing laboratory and anthropometric measures related to diagnosis of MetS during follow up $(\mathrm{n}=309)$ and participants with undetermined genotype $(n=485)$ were excluded from the study. The final analysis was conducted based on the data from 1423 participants, from 2006 until 2018, during the 8.9-year follow-up (Fig. 1).

This study protocol has been approved by the ethics committee of the Research Institute for Endocrine Sciences (RIES), Shahid Beheshti University of Medical Sciences (No. IR.SBMU.ENDOCRINE.REC.1398.084), and written informed consent was obtained from all participants. 


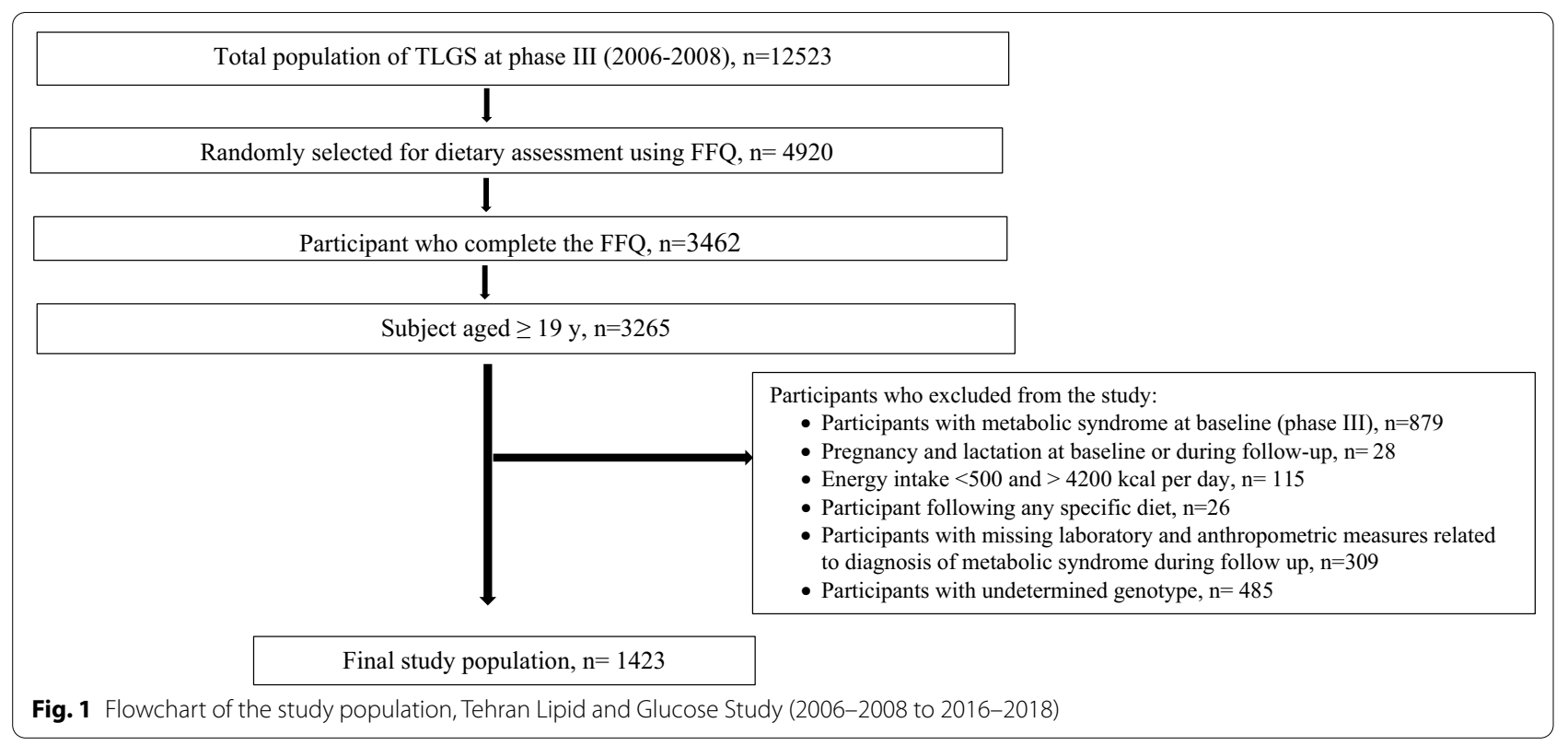

\section{Dietary assessment}

A validated, semi-quantitative food frequency questionnaire (FFQ) was used to obtain and update dietary intake information every 3 years [30]. The primary FFQ had 168 food items in 2006 then the food items were decreased to 147 in 2009, due to the low frequency of consumption of certain food items and combining selected food items. Consumption frequencies of each food item on a daily, weekly, or monthly basis during the previous year were documented according to a standard unit or portion size, evaluated in household measures specified for each food, during face-to-face interviews by expert dietitians, and consumed portion sizes were converted to grams. Since the Iranian food composition table (FCT) is incomplete, the US Department of Agriculture (USDA) FCT (https ://ndb.nal.usda.gov/ndb/ accessed 15 March 2019) was used to calculate energy, macro- and micronutrients. Total energy, macro- and micronutrient content were calculated by summing up consumption of these nutrients. Due to the crucial impact of recent dietary intakes on the association of diet and chronic disease, in the present study we used an alternative approach, providing more weight to the recent diet, to reduce within subject variations [31]. From the initial number of 1423 participants at baseline, 453 participants completed all 4 FFQs, 499 participants completed 3 FFQs, 278 participants completed 2 FFQs, and 193 participants did not complete the any FFQs during follow-up. To impute missing values, last observation carried forward method was used [31].

Nut consumption collected using the FFQ showed a valid estimate against multiple dietary records. A good correlation coefficient was observed between FFQ and multiple 24 recalls $(0.54$ and 0.39 in males and females, respectively) and between two FFQs (0.34 and 0.52 in males and females, respectively) [32]. Moreover, there was reasonable reliability, validity and stability of the dietary patterns, using data from the FFQ, over the 8-year follow-up period [33].

\section{Biochemical assessment}

At baseline and follow-up, between 7:00 and 9:00 a.m., venous blood samples were obtained from all study participants who fasted for $12-14 \mathrm{~h}$ over the previous night. The blood samples were placed into vacutainer tubes, and were centrifuged within 30-45 min of collection. Fasting blood glucose (FPG), high density lipoprotein (HDL-C), and triglyceride (TG) analysis were done at the TLGS research laboratory on the day of sample collection, using a selector 2 auto-analyzer (Vital Scientific, Spankeren, the Netherlands) and commercial kits (Pars Azmoon Inc., Tehran, Iran). FPG was analyzed using an enzymatic colorimetric method with the glucose oxidase technique; inter- and intra-assay coefficients of variation (CV) at baseline and followup phases were both less than $<2.3 \%$. TG was assayed using an enzymatic colorimetric method with glycerol phosphate oxidase. HDL-C was analyzed after precipitation of apolipoprotein B-containing lipoproteins with phosphotungstic acid. Both intra- and inter-assay CVs were below 2.1 and $3.0 \%$ for TG and HDL-C, respectively, in all baseline and follow-up assays. All samples were analyzed when internal quality control met the acceptable criteria. 


\section{Assessment of other variables}

At the baseline recruitment, data on characteristics of participants were collected by trained researchers using a standardized questionnaire, which included demographic information, lifestyle factors (smoking status and physical activity), socioeconomic status (education, and employment), medication regimen (antihypertensive, lipid-lowering and anti-diabetic agents), past medical history. Details of physical assessment including weight, height, waist circumference, and blood pressure have been documented elsewhere [28]. Briefly, participants' weight, while being minimally clothed without wearing shoes, was measured using a digital scale (Seca 707: range $0-150 \mathrm{~kg}$ ) and recorded with accuracy of $100 \mathrm{~g}$. Height was measured in a standing position, with shoulders in normal alignment, without shoes, using Seca 225 stadiometer (Seca $\mathrm{GmbH}$ ) and recorded to the nearest $0.5 \mathrm{~cm}$. BMI was calculated by dividing weight in kilograms into height per square meter. Waist circumference (WC) was measured at the umbilical level using an un-stretched tape measure, without any pressure to the body surface, and was recorded to the accuracy of $0.5 \mathrm{~cm}$. After participants rested in a sitting position for $15 \mathrm{~min}$, blood pressure was measured using a standardized mercury sphygmomanometer (calibrated by the Iranian Institute of Standards and Industrial Researches) on the right arm twice at least $30 \mathrm{~s}$ apart, and the average of the two measurements was reported as the blood pressure. Physical activity was assessed using modifiable activity questionnaire (MAQ) that included a list all three forms of activities, including leisure time, job, and household activities; the frequency and amount of time spent per week engaged in physical activity over the last year were recorded [34]. Physical activity levels were expressed as metabolic equivalent hours per week (METsh/week). The reliability and convergent validity of the Persian version of the MAQ had been reported [35].

\section{Definition of metabolic syndrome}

In accordance with the Joint Interim Statement, diagnosis of MetS required the presence of three or more of the following criteria [1]: (1) elevated glucose concentrations (FPG concentration $\geq 100 \mathrm{mg} / \mathrm{dL}$ or treatment with anti-hyperglycemic medications), (2) TG concentration $(\geq 150 \mathrm{mg} / \mathrm{dL}$ or treatment with anti-hypertriglyceridemia medications), (3) reduced serum HDL-C (<50 mg/ $\mathrm{dL}$ in women and $<40 \mathrm{mg} / \mathrm{dL}$ in men), (4) elevated blood pressure $(\geq 130 / 85 \mathrm{mmHg}$ or treatment with anti-hypertensive medications), and (5) enlarged abdominal circumference ( $\geq 95 \mathrm{~cm}$ according to the population- and country-specific cut-off points for Iranian adults of both genders) [36].

\section{Genotyping}

Genomic DNA was extracted from the buffy-coat of samples using a proteinase $\mathrm{K} /$ salting out standard method [37]. The quality of extracted DNA was determined using the Nanodrop 1000 Spectrophotometer. Samples in the range of $1.7<\mathrm{A} 260 / \mathrm{A} 280<2$ were excluded due to low quality and concentration.

DNA samples were processed on HumanOmniExpress-24-v1-0 bead chips (containing 649,932 SNP loci with an average mean distance of $4 \mathrm{~kb}$ ) at the deCODE genetics company (Reykjavik, Iceland) according to the manufacturer's specifications (Illumina Inc., San Diego, CA, USA). Quality control procedures performed by PLINK program (V 1.07) and R statistic (V 3.2) [38]. Then the genotyping data of two TCF7L2 polymorphisms (rs7903146 and rs12255372) was used for association analysis. In our study population, the TCF7L2 SNPs (rs12255372 and rs7903146) showed moderate linkage disequilibrium $\left(\mathrm{r}^{2}=0.67\right)$.

\section{Statistical analysis}

Statistical analyses were performed using SPSS package, version 17.0 (SPSS, Chicago, IL). All tests were twotailed, and P values $<0.05$ were considered as statistically significant. The significance of deviations of observed genotype frequencies from those predicted by the Hardy-Weinberg equation were evaluated with $\chi^{2}$ test. Based on TCF7L2 rs7903146 and rs12255372 genotypes, participants were categorized according to major allele homozygotes, heterozygotes, and minor allele homozygotes (additive model). Chi-square for categorical data and general linear models for continuous data were used to compared baseline characteristics and dietary variables across tertiles of nut consumption. Hazard ratio (95\% confidence interval) was computed using Cox proportional hazards regression analyses. The first model was crude. The second model was adjusted for age, gender, smoking, physical activity, education levels, occupational status, total energy intake, fiber intake, family history of diabetes, family history of cardiovascular disease. Model 3 was further adjusted for BMI at baseline. Regarding the interaction between weight change during follow-up and rs7903146 and rs12255372 genotypes (three categories) on the risk of MetS, we stratified participants according to their weight change $(<7 \%$ and $\geq 7 \%)$ and investigated the effect modification of weight change on the association between nut consumption and risk of MetS based on rs7903146 and rs12255372 genotypes.

\section{Results}

Genotype distributions were in Hardy-Weinberg equilibrium ( $\mathrm{P}=0.50$ for $\mathrm{rs} 12255372$ and $\mathrm{P}=0.27$ for rs7903146). Considering the rs7903146 genotypes, the 
frequency of $\mathrm{CT}$ and $\mathrm{TT}$ were $46.5 \%$ and $14.7 \%$, respectively, whereas the frequency of GT and TT genotypes were $44.9 \%$ and $13.0 \%$ in rs 12255372 . Genotype effect on MetS risk found for both rs7903146 and rs12255372 genotypes after adjustment for age, gender and BMI (Table 1). Participants with the CT and TT genotypes of the rs7903146 had a HR of 1.21 (0.97-1.49) and 1.47 (1.10-1.95) for incident MetS, compared with CC genotype, whereas HR (95\% CIs) of MetS were 1.21 (0.981.49) and 1.47 (1.09-1.98) for those with the rs 12255372 GT and TT genotypes, respectively.

We documented 415 new cases of MetS. The median nut consumption was $20.0 \mathrm{~g} /$ week (IQR: 8.6-38.9 g/ week). Baseline characteristics of the study participants based on both rs7903146 genotype and tertiles of nut consumption are presented in Table 2. There was no significant difference in age, gender, physical activity, occupation status, family history of diabetes, family history of CVD, and BMI across tertiles of nut consumption in rs7903146 SNP. Participants in the highest tertile of nuts intake who were carries of rs7903146 $\mathrm{T}$ allele were less likely to be smokers and those with CC genotype was more educated. Participants in highest tertile of nut consumption were more likely to have higher energy intakes, dietary fiber, fruit, and vegetable intakes. Additionally, participants with CC genotype who were in the highest tertile of nuts intake consumed more dairy products, poultry, fish, Meat, processed meat and organ meat and legume. Participants with CT genotype consumed more dairy products and dietary cholesterol and participants with TT genotype consumed more dietary cholesterol, whole grain, refined grain, legume and dairy products.

Table 3 presents the multivariable-adjusted HR for the MetS risk across tertiles of nut consumption according to TCF7L2 rs7903146 and rs12255372 genotype, in total population. Regarding the rs7903146 variant, in participants with CT genotype, highest tertile of nut consumption was associated with a $36 \%$ reduction in the risk of MetS in crude model (HR: 0.64, CI (0.45-0.90)), this association remained significant after further adjustments in model 2 and 3 (HR: 0.61, CI (0.43-0.87) and HR: 0.63 , CI (0.44-0.90), respectively). Additionally, carriers of T-allele $(\mathrm{CT}+\mathrm{TT})$ in the third tertile of nut consumption had a significantly lower risk of MetS in all 3 statistical models, a 33\% reduction of risk in crude model, a $34 \%$ reduction of risk in model 2 and a 33\% reduction in model 3 (HR: 0.67, CI (0.50-0.90), HR: 0.66, CI (0.49-0.89), and HR: 0.67, CI (0.50-0.91), respectively). As for the rs 12255372 variant, highest versus lowest tertile of nut consumption in participants with GT genotype resulted in a $32 \%$ and $33 \%$ reduction of MetS risk in model 2 and model 3 (HR: 0.68, CI (0.48-0.96) and HR: 0.67 , CI (0.47-0.95), respectively). In individuals with TT genotype, third tertile of nut consumption was associated with a lower risk of MetS in model 1, this association diminished to non-significant levels after further adjustments. Moreover, in carriers of T-allele $(\mathrm{CT}+\mathrm{TT})$ consumption of nuts significantly reduced the risk of MetS in all 3 models.

An interaction of MetS risk was found for nut consumption, TCF7L2 variants, and weigh change during follow-up. In participants with $<7 \%$ weight gain during the follow-up, the multivariable-adjusted HRs (95\% CI) for the MetS risk across tertiles of nut consumption according to both TCF7L2 rs7903146 and rs12255372 genotypes are presented in Table 4. In the rs12255372 GT genotype, third tertile of nuts intake reduced the risk of MetS by $47 \%$ in model 2 (HR: 0.53 , CI (0.30-0.94). At last, GT + TT individuals in the highest tertile of nut consumption had a $46 \%$ reduction

Table 1 Genotypes and allele frequency of TCF7L2 SNPs

\begin{tabular}{|c|c|c|c|c|c|c|c|c|c|c|}
\hline & \multicolumn{2}{|l|}{ CC } & \multicolumn{2}{|l|}{ CT } & \multicolumn{2}{|l|}{ TT } & \multicolumn{2}{|c|}{ C allele frequency } & \multicolumn{2}{|c|}{ T allele frequency } \\
\hline & Non-MetS & MetS & Non-MetS & MetS & Non-MetS & MetS & Non-MetS & MetS & Non-MetS & MetS \\
\hline \multicolumn{11}{|l|}{ Rs7903146 } \\
\hline Total population & 407 & 145 & 461 & 200 & 140 & 70 & 1275 & 490 & 741 & 340 \\
\hline Weigh change $<7 \%$ & 234 & 58 & 309 & 92 & 93 & 18 & 777 & 208 & 495 & 128 \\
\hline \multirow[t]{3}{*}{ Weight change $\geq 7 \%$} & 173 & 87 & 152 & 108 & 47 & 52 & 498 & 282 & 246 & 212 \\
\hline & \multicolumn{2}{|l|}{ GG } & \multicolumn{2}{|l|}{ GT } & \multicolumn{2}{|l|}{ TT } & \multicolumn{2}{|c|}{ G allele frequency } & \multicolumn{2}{|c|}{$\mathrm{T}$ allele frequency } \\
\hline & Non-MetS & MetS & Non-MetS & MetS & Non-MetS & MetS & Non-MetS & MetS & Non-MetS & MetS \\
\hline \multicolumn{11}{|l|}{ Rs12255372 } \\
\hline Total population & 440 & 159 & 443 & 196 & 125 & 60 & 1323 & 514 & 693 & 316 \\
\hline Weigh change < 7\% & 255 & 67 & 296 & 82 & 85 & 19 & 806 & 216 & 466 & 120 \\
\hline Weight change $\geq 7 \%$ & 185 & 92 & 147 & 114 & 40 & 41 & 517 & 298 & 227 & 196 \\
\hline
\end{tabular}

Number of participants 
Table 2 Baseline characteristics and intake of dietary variables of study population across tertiles of nut consumption according to transcription factor-7-like 2 rs7903146 genotype

\begin{tabular}{|c|c|c|c|c|c|c|c|c|c|}
\hline \multirow[t]{2}{*}{ rs7903146 genotype } & \multicolumn{2}{|l|}{$\mathrm{CC}$} & \multirow[t]{2}{*}{ P value } & \multicolumn{2}{|l|}{ CT } & \multirow[t]{2}{*}{ P value } & \multicolumn{2}{|l|}{ TT } & \multirow[t]{2}{*}{$P$ value } \\
\hline & $\mathrm{T} 1$ & T3 & & $\mathrm{T} 1$ & T3 & & T1 & T3 & \\
\hline Age (y) & $35.2 \pm 0.9$ & $37.7 \pm 0.9$ & 0.16 & $37.6 \pm 0.8$ & $37.9 \pm 0.8$ & 0.62 & $35.0 \pm 1.6$ & $35.7 \pm 1.6$ & 0.82 \\
\hline Female (\%) & 61.5 & 55.7 & 0.41 & 40.7 & 36.0 & 0.49 & 53.5 & 65.7 & 0.33 \\
\hline Physical activity (MET h-week) & $4.3 \pm 0.3$ & $4.2 \pm 0.3$ & 0.45 & $4.3 \pm 0.3$ & $4.4 \pm 0.3$ & 0.58 & $4.2 \pm 0.3$ & $4.3 \pm 0.3$ & 0.51 \\
\hline Current smoking (\%) & 21.5 & 19.6 & 0.63 & 22.6 & 15.1 & 0.04 & 29.6 & 14.3 & 0.05 \\
\hline Academic degrees (\%) & 19.5 & 33.0 & 0.01 & 20.4 & 24.9 & 0.14 & 26.8 & 22.9 & 0.48 \\
\hline Occupational status, employed (\%) & 41.0 & 45.6 & 0.46 & 40.4 & 43.4 & 0.72 & 50.7 & 32.9 & 0.08 \\
\hline Family history of diabetes (\%) & 33.3 & 37.6 & 0.76 & 35.4 & 28.9 & 0.62 & 32.4 & 28.6 & 0.08 \\
\hline Family history of CVD events (\%) & 16.9 & 16.0 & 0.73 & 38.9 & 40.9 & 0.98 & 25.4 & 10.0 & 0.08 \\
\hline BMI at baseline $\left(\mathrm{kg} / \mathrm{m}^{2}\right)$ & $25.7 \pm 0.3$ & $25.9 \pm 0.3$ & 0.89 & $25.7 \pm 0.3$ & $26.0 \pm 0.3$ & 0.11 & $25.7 \pm 0.5$ & $25.0 \pm 0.5$ & 0.64 \\
\hline \multicolumn{10}{|l|}{ Dietary variables } \\
\hline Total energy (Kcal/d) & $2123 \pm 87$ & $2562 \pm 88$ & 0.002 & $2106 \pm 44$ & $2460 \pm 44$ & $<0.001$ & $2181 \pm 81$ & $2640 \pm 81$ & $<0.001$ \\
\hline Carbohydrate (\% of total energy) & $60.3 \pm 1.0$ & $61.8 \pm 1.0$ & 0.17 & $61.2 \pm 0.7$ & $61.7 \pm 0.7$ & 0.82 & $059.5 \pm 1.2$ & $61.5 \pm 1.2$ & 0.43 \\
\hline Protein (\% of total energy) & $14.3 \pm 0.9$ & $14.5 \pm 0.9$ & 0.23 & $14.5 \pm 0.2$ & $14.6 \pm 0.2$ & 0.19 & $14.0 \pm 0.3$ & $14.5 \pm 0.3$ & 0.06 \\
\hline Fat (\% of total energy) & $30.8 \pm 0.4$ & $30.5 \pm 0.4$ & 0.13 & $29.6 \pm 0.3$ & $30.0 \pm 0.3$ & 0.69 & $31.0 \pm 0.6$ & $30.4 \pm 0.6$ & 0.75 \\
\hline SFA (\% of total energy) & $10.3 \pm 0.2$ & $9.7 \pm 0.2$ & 0.11 & $9.9 \pm 0.2$ & $9.6 \pm 0.2$ & 0.44 & $9.7 \pm 0.3$ & $9.6 \pm 0.3$ & 0.22 \\
\hline MUFA (\% of total energy) & $10.7 \pm 0.3$ & $10.2 \pm 0.3$ & 0.47 & $10.1 \pm 0.1$ & $10.1 \pm 0.1$ & 0.98 & $10.8 \pm 0.3$ & $10.1 \pm 0.3$ & 0.13 \\
\hline PUFA (\% of total energy) & $6.2 \pm 0.1$ & $6.3 \pm 0.1$ & 0.32 & $5.9 \pm 0.1$ & $6.2 \pm 0.1$ & 0.14 & $6.6 \pm 0.2$ & $6.2 \pm 0.2$ & 0.11 \\
\hline Total fiber (g/d) & $37.9 \pm 1.2$ & $47.2 \pm 1.2$ & $<0.001$ & $39.2 \pm 1.2$ & $46.1 \pm 1.2$ & $<0.001$ & $39.6 \pm 2.2$ & $49.8 \pm 2.2$ & $<0.001$ \\
\hline Cholesterol (g/d) & $200 \pm 24$ & $258 \pm 24$ & 0.07 & $209 \pm 7.5$ & $240 \pm 7.6$ & 0.018 & $202 \pm 12$ & $255 \pm 12$ & 0.013 \\
\hline Vegetable (g/d) & $247 \pm 11$ & $338 \pm 11$ & $<0.001$ & $247 \pm 9$ & $320 \pm 9$ & $<0.001$ & $246 \pm 15$ & $333 \pm 15$ & $<0.001$ \\
\hline Fruit (g/d) & $279 \pm 18$ & $497 \pm 18$ & $<0.001$ & $303 \pm 20$ & $472 \pm 20$ & $<0.001$ & $270 \pm 32$ & $510 \pm 32$ & $<0.001$ \\
\hline Meat, processed meat and organ meat(g/d) & $23.9 \pm 0.3$ & $33.3 \pm 1.3$ & $<0.001$ & $24.8 \pm 1.4$ & $28.4 \pm 1.4$ & 0.12 & $24.3 \pm 2.5$ & $31.7 \pm 2.5$ & 0.09 \\
\hline Poultry and fish (g/d) & $33.0 \pm 2.9$ & $46.8 \pm 2.9$ & $<0.001$ & $35.5 \pm 6$ & $150 \pm 6$ & 0.06 & $32.3 \pm 3.8$ & $44.5 \pm 3.9$ & 0.06 \\
\hline Whole grain $(\mathrm{g} / \mathrm{d})$ & $137 \pm 6$ & $151 \pm 7$ & 0.37 & $139 \pm 6$ & $150 \pm 6$ & 0.25 & $115 \pm 9.8$ & $158 \pm 9.9$ & 0.01 \\
\hline Refined grain (g/day) & $336 \pm 12$ & $315 \pm 12$ & 0.43 & $322 \pm 9$ & $314 \pm 9$ & 0.31 & $378 \pm 19$ & $342 \pm 19$ & 0.04 \\
\hline Legumes (g/d) & $31.0 \pm 1.8$ & $39.8 \pm 1.8$ & 0.001 & $35.9 \pm 1.6$ & $39.6 \pm 1.6$ & 0.10 & $35.3 \pm 3.9$ & $50.1 \pm 3.9$ & 0.007 \\
\hline Dairy products (g/d) & $353 \pm 16$ & $420 \pm 16$ & 0.013 & $344 \pm 14$ & $410 \pm 14$ & 0.004 & $336 \pm 25$ & $435 \pm 26$ & 0.02 \\
\hline
\end{tabular}

Data are express as mean $\pm \mathrm{SE}$, unless otherwise indicated

Dietary data were adjusted for $\mathrm{kcal}$

MetS risk in model 2 (HR: 0.54, CI (0.32-0.90). No association was found between nut consumption and risk of MetS among rs7903146 genotypes. Table 5 presents the multivariable-adjusted HRs (95\% CI) for the MetS risk across tertiles of nut consumption according to both TCF7L2 rs7903146 and rs12255372 genotype among participant with a weight gain $\geq 7 \%$. Regarding rs7903146, in subjects with CT genotype, nut consumption was associated with a lower risk of MetS after adjustment for cofounding factors (highest vs. lowest tertile of consumption: HR: 0.49 , CI $(0.30-0.79)$ ). $\mathrm{CT}+\mathrm{TT}$ individuals in the highest tertile of nut consumption had a $45 \%$ reduction MetS risk after adjustment for confounding factors (HR: 0.55, CI (0.37-0.82). No association was found between nut consumption and risk of MetS among rs12255372 genotypes.

\section{Discussion}

In this population-based prospective study, the protective effect of higher nut consumption against the MetS incidence depended on individuals' genetic background. In our total population, higher nut consumption reduced risk of MetS among carriers of a single T-risk allele of the TCF7L2 rs7903146 and rs12255372 variants. In rs7903146 variant, CT $+\mathrm{TT}$ individuals with high weight gain experienced a lower risk of MetS; however, considering rs12255372 genotype, higher nut consumption reduced risk of MetS only among participants with low weight gain.

With respect to gene-diet associations, previous prospective cohort and interventional studies have reported modifying effects of the TCF7L2 polymorphisms on the diet-disease associations [9, 11-13, 
Table 3 Hazard ratios of metabolic syndrome across tertiles of nut consumption according to transcription factor-7-like 2 rs7903146 and rs 12255372 genotypes

\begin{tabular}{llll}
\hline T1 & T2 & T3 & $\mathrm{P}_{\text {trend }}{ }^{\mathrm{a}}$
\end{tabular}

Rs7903146

CC

$\begin{array}{lllll}\text { Model 1 } & 1 & 0.95(0.63-1.42) & 0.97(0.65-1.45) & 0.97 \\ \text { Model 2 } & 1 & 0.88(0.58-1.33) & 0.84(0.55-1.27) & 0.71 \\ \text { Model 3 } & 1 & 0.85(0.56-1.29) & 0.82(0.54-1.25) & 0.63 \\ \text { CT } & & & & \end{array}$

\begin{tabular}{|c|c|c|c|c|}
\hline Model 1 & 1 & $0.71(0.51-0.99)$ & $0.64(0.45-0.90)$ & 0.02 \\
\hline Model 2 & 1 & $0.67(0.47-0.94)$ & $0.61(0.43-0.87)$ & 0.01 \\
\hline Model 3 & 1 & $0.70(0.49-0.99)$ & $0.63(0.44-0.90)$ & 0.02 \\
\hline \multicolumn{5}{|l|}{ TT } \\
\hline Model 1 & 1 & $0.80(0.46-1.39)$ & $0.61(0.34-1.11)$ & 0.27 \\
\hline Model 2 & 1 & $0.79(0.44-1.41)$ & $0.64(0.35-1.18)$ & 0.36 \\
\hline Model 3 & 1 & $0.78(0.43-1.42)$ & $0.72(0.39-1.34)$ & 0.56 \\
\hline \multicolumn{5}{|l|}{$\mathrm{CT}+\mathrm{TT}$} \\
\hline Model 1 & 1 & $0.73(0.55-0.97)$ & $0.67(0.50-0.90)$ & 0.01 \\
\hline Model 2 & 1 & $0.70(0.52-0.93)$ & $0.66(0.49-0.89)$ & 0.01 \\
\hline Model 3 & 1 & $0.72(0.54-0.97)$ & $0.67(0.50-0.91)$ & 0.02 \\
\hline \multicolumn{5}{|l|}{ Rs 12255372} \\
\hline \multicolumn{5}{|l|}{ GG } \\
\hline Model 1 & 1 & $0.94(0.64-1.38)$ & $1.04(0.71-1.52)$ & 0.86 \\
\hline Model 2 & 1 & $0.83(0.55-1.24)$ & $0.89(0.60-1.32)$ & 0.65 \\
\hline Model 3 & 1 & $0.80(0.53-1.19)$ & $0.92(0.62-1.37)$ & 0.54 \\
\hline \multicolumn{5}{|l|}{ GT } \\
\hline Model 1 & 1 & $0.78(0.56-1.10)$ & $0.77(0.55-1.08)$ & 0.18 \\
\hline Model 2 & 1 & $0.74(0.52-1.04)$ & $0.68(0.48-0.96)$ & 0.04 \\
\hline Model 3 & 1 & $0.75(0.53-1.07)$ & $0.67(0.47-0.95)$ & 0.03 \\
\hline \multicolumn{5}{|l|}{$\mathrm{TT}$} \\
\hline Model 1 & 1 & $0.53(0.29-0.98)$ & $0.49(0.26-0.91)$ & 0.03 \\
\hline Model 2 & 1 & $0.58(0.31-1.09)$ & $0.63(0.32-1.26)$ & 0.18 \\
\hline Model 3 & 1 & $0.66(0.34-1.26)$ & $0.70(0.35-1.39)$ & 0.39 \\
\hline \multicolumn{5}{|l|}{$\mathrm{GT}+\mathrm{TT}$} \\
\hline Model 1 & 1 & $0.69(0.51-0.93)$ & $0.69(0.51-0.93)$ & 0.03 \\
\hline Model 2 & 1 & $0.68(0.51-0.92)$ & $0.66(0.49-0.90)$ & 0.02 \\
\hline Model 3 & 1 & $0.69(0.51-0.93)$ & $0.66(0.49-0.69)$ & 0.01 \\
\hline
\end{tabular}

Model 1 was crude

Model 2 was adjusted for age, gender, smoking, physical activity, education levels, occupational status, total energy intake, fiber intake, family history of diabetes, family history of cardiovascular disease

Model 3 was additionally adjusted for BMI at baseline

a The median intake of each tertile category was assigned and then these quartile median variables were included as a continuous variable in cox proportional hazards regression

39-42]. In line with our findings suggesting risk-lowering effects of nut consumption on MetS in participants with genotypes CT + TT rs7903146 and GT + TT
Table 4 Hazard ratios of metabolic syndrome across tertiles of nut consumption according to transcription factor-7-like 2 rs7903146 and rs12255372 genotype among participant with weigh change $<7 \%$ during follow-up

\begin{tabular}{|c|c|c|c|c|}
\hline & \multicolumn{4}{|c|}{ Tertiles of nut consumption } \\
\hline & $\mathrm{T} 1$ & $\mathrm{~T} 2$ & T3 & $P_{\text {trend }}{ }^{a}$ \\
\hline \multicolumn{5}{|l|}{ Rs7903146 } \\
\hline \multicolumn{5}{|l|}{$\mathrm{CC}$} \\
\hline Model 1 & 1 & $0.80(0.42-1.50)$ & $0.86(0.46-1.62)$ & 0.78 \\
\hline Model 2 & 1 & $0.76(0.39-1.46)$ & $0.69(0.35-1.36)$ & 0.53 \\
\hline \multicolumn{5}{|l|}{$\mathrm{CT}$} \\
\hline Model 1 & 1 & $0.76(0.48-1.31)$ & $0.85(0.52-1.39)$ & 0.73 \\
\hline Model 2 & 1 & $0.81(0.49-1.34)$ & $0.72(0.42-1.21)$ & 0.32 \\
\hline \multicolumn{5}{|l|}{$\mathrm{TT}$} \\
\hline Model 1 & 1 & $0.69(0.24-1.99)$ & $0.46(0.13-1.54)$ & 0.44 \\
\hline Model 2 & 1 & $0.51(0.16-1.61)$ & $0.35(0.09-1.36)$ & 0.27 \\
\hline \multicolumn{5}{|l|}{$\mathrm{CT}+\mathrm{TT}$} \\
\hline Model 1 & 1 & $0.73(0.46-1.14)$ & $0.75(0.48-1.18)$ & 0.30 \\
\hline Model 2 & 1 & $0.74(0.46-1.17)$ & $0.63(0.39-1.02)$ & 0.13 \\
\hline \multicolumn{5}{|c|}{ Rs 12255372} \\
\hline \multicolumn{5}{|l|}{ GG } \\
\hline Model 1 & 1 & $0.66(0.36-1.25)$ & 1.07 (0.61-1.88) & 0.29 \\
\hline Model 2 & 1 & $0.70(0.36-1.35)$ & $0.89(0.47-1.65)$ & 0.40 \\
\hline \multicolumn{5}{|l|}{ GT } \\
\hline Model 1 & 1 & $0.92(0.55-1.54)$ & $0.70(0.41-1.21)$ & 0.19 \\
\hline Model 2 & 1 & $0.85(0.50-1.43)$ & $0.53(0.30-0.94)$ & 0.02 \\
\hline \multicolumn{5}{|l|}{$\mathrm{TT}$} \\
\hline Model 1 & 1 & $0.58(0.21-1.65)$ & $0.40(0.12-1.30)$ & 0.15 \\
\hline Model 2 & 1 & $0.63(0.21-1.89)$ & $0.95(0.21-4.20)$ & 0.68 \\
\hline \multicolumn{5}{|l|}{$\mathrm{GT}+\mathrm{TT}$} \\
\hline Model 1 & 1 & $0.81(0.51-1.29)$ & $0.66(0.41-1.07)$ & 0.11 \\
\hline Model 2 & 1 & $0.76(0.48-1.22)$ & $0.54(0.32-0.90)$ & 0.02 \\
\hline
\end{tabular}

Model 1 was crude

Model 2 was adjusted for age, gender, smoking, physical activity, education levels, occupational status, total energy intake, fiber intake, family history of diabetes, family history of cardiovascular disease

a The median intake of each tertile category was assigned and then these quartile median variables were included as a continuous variable in cox proportional hazards regression

rs12255372 risk, data have reported a protective effect of other dietary compounds, as well as dietary patterns, against type 2 diabetes risk in subjects carrying the riskallele $\mathrm{T}$ of the TCF7L2 variants [39-41]. For instance, in a PREDIMED randomized controlled trial [40], adherence to the Mediterranean diet resulted in lower fasting plasma glucose and lipid profile concentrations among TCF7L2 rs7903146 TT genotype. Moreover, after a median 4.8 years of follow-up, the Mediterranean diet attenuated the risk of stroke in TT individuals who were at a higher genetic susceptibility to stroke 


\begin{abstract}
Table 5 Hazard ratios of metabolic syndrome across tertiles of nut consumption according to transcription factor-7-like 2 rs7903146 and rs12255372 genotype among participant with weigh change $\geq 7 \%$ during follow-up
\end{abstract}

\begin{tabular}{|c|c|c|c|c|}
\hline & \multicolumn{4}{|c|}{ Tertiles of nut consumption } \\
\hline & $\mathrm{T} 1$ & $\mathrm{~T} 2$ & T3 & $\mathrm{P}_{\text {trend }}{ }^{\mathrm{a}}$ \\
\hline \multicolumn{5}{|l|}{ Rs7903146 } \\
\hline \multicolumn{5}{|l|}{$\mathrm{CC}$} \\
\hline Model 1 & 1 & $1.21(0.72-2.03)$ & $1.05(0.62-1.78)$ & 0.74 \\
\hline Model 2 & 1 & $1.33(0.77-2.26)$ & $105(0.61-1.81)$ & 0.51 \\
\hline \multicolumn{5}{|l|}{$\mathrm{CT}$} \\
\hline Model 1 & 1 & $0.58(0.37-0.91)$ & $0.54(0.33-0.85)$ & 0.01 \\
\hline Model 2 & 1 & $0.57(0.35-0.92)$ & $0.49(0.30-0.79)$ & 0.007 \\
\hline \multicolumn{5}{|l|}{$\mathrm{TT}$} \\
\hline Model 1 & 1 & $1.14(0.59-2.20)$ & $0.91(0.45-1.83)$ & 0.80 \\
\hline Model 2 & 1 & $1.25(0.58-2.69)$ & $0.69(0.31-1.51)$ & 0.27 \\
\hline \multicolumn{5}{|l|}{$\mathrm{CT}+\mathrm{TT}$} \\
\hline Model 1 & 1 & $0.70(0.48-1.01)$ & $0.59(0.40-0.87)$ & 0.02 \\
\hline Model 2 & 1 & $0.67(0.46-0.97)$ & $0.55(0.37-0.82)$ & 0.009 \\
\hline \multicolumn{5}{|l|}{ Rs 12255372} \\
\hline \multicolumn{5}{|l|}{ GG } \\
\hline Model 1 & 1 & $1.02(0.62-1.71)$ & $1.04(0.63-1.72)$ & 0.98 \\
\hline Model 2 & 1 & $1.06(0.63-1.80)$ & $1.09(0.65-1.82)$ & 0.94 \\
\hline \multicolumn{5}{|l|}{ GT } \\
\hline Model 1 & 1 & $0.58(0.36-1.12)$ & $0.75(0.49-1.16)$ & 0.37 \\
\hline Model 2 & 1 & $0.57(0.35-1.12)$ & $0.74(0.47-1.17)$ & 0.38 \\
\hline \multicolumn{5}{|l|}{$\mathrm{TT}$} \\
\hline Model 1 & 1 & $0.85(0.41-1.77)$ & $0.68(0.31-1.46)$ & 0.34 \\
\hline Model 2 & 1 & $0.60(0.25-1.43)$ & $0.36(0.14-1.10)$ & 0.14 \\
\hline \multicolumn{5}{|l|}{$\mathrm{GT}+\mathrm{TT}$} \\
\hline Model 1 & 1 & $0.60(0.41-1.09)$ & $0.71(0.49-1.03)$ & 0.16 \\
\hline Model 2 & 1 & $0.60(0.45-1.09)$ & $0.71(0.49-1.03)$ & 0.10 \\
\hline
\end{tabular}

Model 1 was crude

Model 2 was adjusted for age, gender, smoking, physical activity, education levels, occupational status, total energy intake, fiber intake, family history of diabetes, family history of cardiovascular disease

a The median intake of each tertile category was assigned and then these quartile median variables were included as a continuous variable in cox proportional hazards regression

[40]. In addition, regarding dietary compounds with detrimental effects on health, a prospective study has documented that dietary fatty acids' type and amount of consumption could potentially exacerbate the risk of developing MetS in individuals with high genetic susceptibility, as reported in the study, high consumption of SFAs and low consumption of polyunsaturated fatty acids (PUFAs) increased the MetS risk in rs7903146 T allele carriers [13]. On the contrary, some of the existing evidence has suggested the protective effects of dietary compounds, such as dietary fiber and cereals, on type 2 diabetes risk among individuals with $\mathrm{C}$ homozygote genotype of the TCF7L2. As demonstrated by the EPIC-Potsdam case-cohort study, the inverse association of whole-grain intake and type 2 diabetes risk was only present in the rs7903146 C homozygote individuals, namely, the $\mathrm{T}$-allele carriers could not benefit from the risk-reducing effects of whole-grains [9]. Similarly, a cross-sectional analysis of the Malmö Diet and Cancer Study Cardiovascular Sub-cohort, indicated that a higher fiber intake was associated with a reduced risk of type 2 diabetes and lower HbA1C levels in individuals with the CC genotype of the TCF7L2 rs7903146 variant; however, individuals carrying T-risk allele lacked this protective effect [11]. Furthermore, results from the Stockholm Diabetes Prevention Program resembled the above-mentioned findings, preventive effects of whole-grains and cereals against developing type 2 diabetes in individuals with the non-risk $\mathrm{C}$ allele, with no observed associations in carriers of the $\mathrm{T}$ risk-allele [42].

$\mathrm{BMI}$, as a main confounder, modulates the association of nut consumption and hazard of chronic disease [25]. In the current study, long-term weight gain of participants modified the effects of nut consumption on the MetS risk, based on rs12255372 and rs7903146 polymorphisms of the TCF7L2 gene, suggesting an interplay of BMI and genetic susceptibility on the association of nut consumption and MetS incidence. The effect of TCF7L2 variants on risk of type 2 diabetes and obesity as well as modulation effect of BMI on the association between diabetes and TCF7L2 variants, have been controversial [26, $27,43]$. This discrepancy, at least in part, may be attributed to the extent of linkage disequilibrium in different population [8, 44]. In the Diabetes Prevention Program, both rs12255373 and rs7903146 predicted progression to diabetes [7], whereas in the Finnish Diabetes Prevention Study only rs 12255372 was significantly associated with the risk of diabetes [8]. In European population, TCF7L2 has not been a risk factor for obesity, but obesity modulates the association between TCf7l2 and risk of type 2 diabetes, additionally, the rs7903146 $\mathrm{T}$ allele was more associated with T2D in non-obese individuals than in obese subjects [45].

Moreover, findings regarding the interaction between dietary determinants and TCF7L2 variants (rs12255372 and rs7903146) on anthropometric measures are controversial. In Tuebingen Lifestyle Intervention Program (TULIP), reduction in BMI and adiposity were observed for both common genotypes of rs12255372 and rs7903146 [46]. In addition, Mediterranean diet significantly interacted with 2 SNPs (rs122255372 and rs7903146) on anthropometric measures; higher Mediterranean score associated with lower BMI and weight 
among risk allele carriers participants [41]. In contrast, in a randomized control trial (RCT), consumption of low fat diet in overweight individuals with risk allele of rs12255372 reduced BMI and fat mass; however, no interaction was found between TCF7L2 rs7903146 and dietary intervention on BMI and adiposity [47]. In another RCT, consumption of wheat bread and nopal tortilla diet, high in dietary fiber, decreased BMI in both CC and CT/TT genotypes of the TCF7L2 rs7903146 variant, yet no significant effect of rs12255372-T risk allele on anthropometric and metabolic indicators was observed [48]. In addition, an interaction between TCF7L2 SNP rs12255372, but not rs7903146, and fiber intake (g/day) on HDL-C was reported [49].

In the current study, we found that nut consumption have greater risk reducing effects on MetS among $\geq 7$ weight gain group with genotype rs7903146, while this risk reduction was obvious among participants with $<7$ weight gain group with rs12255372 genotype. In addition, this effect was only observed among participants with heterozygote $\mathrm{T}$ genotypes. Lack of observed association between nut consumption and risk of MetS among homozygote $\mathrm{T}$ carriers may be due to lower sample size in this population, thereby having insufficient power to detect an association; a major problem that was shown in genetic associating studies [50]. In the current study statistical power for detection of risk of MetS among TT genotypes of the TCF7L2 rs7903146 and rs12255372 variants in total population (0.145-0.464) as well as based on weight changes $(0.143-0.756)$ during follow-up was low; suggesting that due to low power, it is not possible to get a definitive result. Therefore our finding needed to be repeated in other ethnic population with different allele frequency, family-based investigations as well as studies with large sample size.

Some strengths and limitations of this study should be acknowledged. First and foremost, its population-based prospective design, use of a validated of $F F Q$, a long enough follow-up, and the availability of data on potential confounders provided strength for this study. However, dietary assessment using FFQ may lead to measurement error, which eventually, places individuals into wrong categorize of intake [51]. As declared before, due to fewer number of individuals with TT genotype, there is possibility that our study lacked the sufficient power to detect an association of nut consumption and MetS risk in this population. Further research with higher sample size with regard to the TCF7L2 risk genotypes is suggested.

\section{Conclusions}

Higher consumption of nuts may reduce the risk of MetS in T-risk allele of the TCF7L2 rs7903146 and rs12255372 variants and weight change may modify this association.

\begin{abstract}
Abbreviations
MetS: Metabolic syndrome; CVD: Cardiovascular disease; SNPs: Single nucleotide polymorphisms; TCF7L2: Transcription factor-7-like 2; SFA: Saturated fatty acids; TLGS: Tehran lipid and glucose study; IQR: Interquartile range; FFQ:

Food frequency questionnaire; RIES: Research Institute for Endocrine Sciences; USDA: US Department of Agriculture; CV: Coefficients of variation; FPG: Fasting blood glucose; HDL-C: High density lipoprotein; TG: Triglyceride; WC: Waist circumference; METsh/week: Metabolic equivalent hours per week; PUFAs: Polyunsaturated fatty acids.
\end{abstract}

\section{Acknowledgements}

We express our appreciation to the participants of this study for their collaboration.

\section{Authors' contributions}

SHN and PM conceptualized and designed the study. SHN, BB, MA analyzed and interpreted the data; SHN, AZ, BB, PM and MF drafted the initial manuscript; FA supervised the project; all authors have read and approved the final version of the manuscript.

\section{Funding}

This work was supported by Shahid Beheshti University of Medical Sciences, Tehran, Iran (Grant 18360).

\section{Availability of data and materials}

The datasets generated and/or analysed during the current study are not publicly available due institution's policy but are available from the corresponding author on reasonable request.

\section{Ethics approval and consent to participate}

The study protocol was approved by the ethics committee of the Research Institute for Endocrine Sciences (RIES), Shahid Beheshti University of Medical Sciences, and written informed consent was acquired from participants prior to their inclusion in the study.

\section{Consent for publication}

Not applicable.

\section{Competing interests}

On behalf of all authors, the corresponding author hereby declares that there is no conflict of interest.

\section{Author details}

${ }^{1}$ Nutrition and Endocrine Research Center, Research Institute for Endocrine Sciences, Shahid Beheshti University of Medical Sciences, Tehran, Iran. ${ }^{2} \mathrm{Cel}-$ lular and Molecular Research Center, Research Institute for Endocrine Sciences, Shahid Beheshti University of Medical Sciences, No 24, A'rabi St, Yeman Av, P.O. Box 19395-4763, Velenjak, Tehran, Iran. ${ }^{3}$ Department of Clinical Nutrition and Dietetics, Faculty of Nutrition Sciences and Food Technology, National Nutrition and Food Technology Research Institute, Shahid Beheshti University of Medical Sciences, No 24, A'rabi St, Yeman Av, P.O. Box 19395-4763, Velenjak, Tehran, Iran. ${ }^{4}$ Endocrine Research Center, Research Institute for Endocrine Sciences, Shahid Beheshti University of Medical Sciences, Tehran, Iran.

Received: 18 May 2020 Accepted: 4 January 2021

Published online: 12 January 2021

\section{References}

1. Alberti KG, Eckel RH, Grundy SM, Zimmet PZ, Cleeman II, Donato KA, et al. Harmonizing the metabolic syndrome: a joint interim statement of the International Diabetes Federation Task Force on Epidemiology and Prevention; National Heart, Lung, and Blood Institute; American Heart Association; World Heart Federation; International Atherosclerosis Society; and International Association for the Study of Obesity. Circulation. 2009;120(16):1640-5. 
2. Guo Y, Musani SK, Sims M, Pearson TA, DeBoer MD, Gurka MJ. Assessing the added predictive ability of a metabolic syndrome severity score in predicting incident cardiovascular disease and type 2 diabetes: the Atherosclerosis Risk in Communities Study and Jackson Heart Study. 2018;10:42.

3. Perez-Martinez P, Phillips CM, Delgado-Lista J, Garcia-Rios A, LopezMiranda J, Perez-Jimenez F. Nutrigenetics, metabolic syndrome risk and personalized nutrition. Curr Vasc Pharmacol. 2013;11(6):946-53.

4. Shiao SPK, Grayson J, Lie A, Yu CH. Personalized Nutrition-Genes, Diet, and Related Interactive Parameters as Predictors of Cancer in Multiethnic Colorectal Cancer Families. Nutrients. 2018;10(6).

5. Kalantari S, Sharafshah A, Keshavarz P, Davoudi A, Habibipour R. Single and multi-locus association study of TCF7L2 gene variants with susceptibility to type 2 diabetes mellitus in an Iranian population. Gene. 2019;696:88-94.

6. Palizban A, Rezaei M, Khanahmad H, Fazilati M. Transcription factor 7-like 2 polymorphism and context-specific risk of metabolic syndrome, type 2 diabetes, and dyslipidemia. J Res Med Sci. 2017;22:40.

7. Florez JC, Jablonski KA, Bayley N, Pollin TI, de Bakker PI, Shuldiner AR, et al. TCF7L2 polymorphisms and progression to diabetes in the Diabetes Prevention Program. N Engl J Med. 2006;355(3):241-50.

8. Wang J, Kuusisto J, Vanttinen M, Kuulasmaa T, Lindstrom J, Tuomilehto J, et al. Variants of transcription factor 7-like 2 (TCF7L2) gene predict conversion to type 2 diabetes in the Finnish Diabetes Prevention Study and are associated with impaired glucose regulation and impaired insulin secretion. Diabetologia. 2007;50(6):1192-200.

9. Fisher E, Boeing $H$, Fritsche A, Doering F, Joost HG, Schulze MB. Wholegrain consumption and transcription factor-7-like 2 (TCF7L2) rs7903146: gene-diet interaction in modulating type 2 diabetes risk. Br J Nutr. 2009;101(4):478-81.

10. Cornelis MC, Qi L, Kraft P, Hu FB. TCF7L2, dietary carbohydrate, and risk of type 2 diabetes in US women. Am J Clin Nutr. 2009;89(4):1256-62.

11. Hindy G, Sonestedt E, Ericson U, Jing XJ, Zhou Y, Hansson O, et al. Role of TCF7L2 risk variant and dietary fibre intake on incident type 2 diabetes. Diabetologia. 2012;55(10):2646-54.

12. Grau K, Cauchi S, Holst C, Astrup A, Martinez JA, Saris WH, et al. TCF7L2 rs 7903146 -macronutrient interaction in obese individuals' responses to a 10-wk randomized hypoenergetic diet. Am J Clin Nutr. 2010;91 (2):472-9.

13. Phillips CM, Goumidi L, Bertrais S, Field MR, McManus R, Hercberg S, et al. Dietary saturated fat, gender and genetic variation at the TCF7L2 locus predict the development of metabolic syndrome. J Nutr Biochem. 2012;23(3):239-44.

14. Qi L, Cornelis MC, Zhang C, van Dam RM, Hu FB. Genetic predisposition, Western dietary pattern, and the risk of type 2 diabetes in men. Am J Clin Nutr. 2009;89(5):1453-8

15. Cai J, Zhang Y, Nuli R, Zhang Y, Abudusemaiti M, Kadeer A, et al. Interaction between dietary patterns and TCF7L2 polymorphisms on type 2 diabetes mellitus among Uyghur adults in Xinjiang Province. China Diabetes Metab Syndr Obes. 2019;12:239-55.

16. Kim Y, Keogh JB, Clifton PM. Benefits of Nut Consumption on Insulin Resistance and Cardiovascular Risk Factors: Multiple Potential Mechanisms of Actions. 2017;9(11).

17. Baik I, Lee M, Jun NR, Lee JY, Shin C. A healthy dietary pattern consisting of a variety of food choices is inversely associated with the development of metabolic syndrome. Nutr Res Pract. 2013;7(3):233-41.

18. Hosseinpour-Niazi S, Hosseini S, Mirmiran P, Azizi F. Prospective Study of Nut Consumption and Incidence of Metabolic Syndrome: Tehran Lipid and Glucose Study. Nutrients. 2017;9(10).

19. Afshin A, Micha R, Khatibzadeh S, Mozaffarian D. Consumption of nuts and legumes and risk of incident ischemic heart disease, stroke, and diabetes: a systematic review and meta-analysis. Am J Clin Nutr. 2014;100(1):278-88

20. SchwingshackI L, Knuppel S, Michels N, Schwedhelm C, Hoffmann G, Iqbal K, et al. Intake of 12 food groups and disability-adjusted life years from coronary heart disease, stroke, type 2 diabetes, and colorectal cancer in 16 European countries. Eur J Epidemiol. 2019.

21. Martinez-Lapiscina EH, Pimenta AM, Beunza JJ, Bes-Rastrollo M, Martinez JA, Martinez-Gonzalez MA. Nut consumption and incidence of hypertension: the SUN prospective cohort. Nutr Metab Cardiovasc Dis. 2010;20(5):359-65.
22. Freisling $\mathrm{H}$, Noh H, Slimani N, Chajes V, May AM, Peeters PH, et al. Nut intake and 5 -year changes in body weight and obesity risk in adults: results from the EPIC-PANACEA study. Eur J Nutr. 2018;57(7):2399-408.

23. Schlesinger S, Neuenschwander M, Schwedhelm C, Hoffmann G, Bechthold A, Boeing $\mathrm{H}$, et al. Food groups and risk of overweight, obesity, and weight gain: a systematic review and dose-response meta-analysis of prospective studies. Adv Nutr. 2019;10(2):205-18.

24. Mazidi M, Vatanparast $H$, Katsiki N, Banach M. The impact of nuts consumption on glucose/insulin homeostasis and inflammation markers mediated by adiposity factors among American adults. Oncotarget. 2018;9(58):31173-86.

25. Liu Z, Wei P, Li X. Is nut consumption associated with decreased risk of type 2 diabetes? Am J Clin Nutr. 2014;100(5):1401-2.

26. Hjort R, Löfvenborg JE, Ahlqvist E, Alfredsson L, Andersson T, Grill V, et al. Interaction between overweight and genotypes of HLA, TCF7L2, and FTO in relation to the risk of Latent Autoimmune Diabetes in Adults and type 2 diabetes. J Clin Endocrinol Metab. 2019;104(10):4815-26.

27. Li L, Wang J, Ping Z, Li Y, Wang C, Shi Y, et al. Interaction analysis of gene variants of TCF7L 2 and body mass index and waist circumference on type 2 diabetes. Clin Nutr. 2020;39(1):192-7.

28. Azizi F, Ghanbarian A, Momenan AA, Hadaegh F, Mirmiran P, Hedayati M, et al. Prevention of non-communicable disease in a population in nutrition transition: Tehran Lipid and Glucose Study phase II. Trials. 2009;10:5.

29. Hosseini-Esfahani F, Jessri M, Mirmiran P, Bastan S, Azizi F. Adherence to dietary recommendations and risk of metabolic syndrome: Tehran Lipid and Glucose Study. Metab Clin Exp 2010;59(12):1833-42.

30. Mirmiran P, Esfahani FH, Mehrabi Y, Hedayati M, Azizi F. Reliability and relative validity of an FFQ for nutrients in the Tehran lipid and glucose study. Public Health Nutr. 2010;13(5):654-62.

31. Hu FB, Stampfer MJ, Rimm E, Ascherio A, Rosner BA, Spiegelman D, et al. Dietary fat and coronary heart disease: a comparison of approaches for adjusting for total energy intake and modeling repeated dietary measurements. Am J Epidemiol. 1999;149(6):531-40.

32. Esfahani FH, Asghari G, Mirmiran P, Azizi F. Reproducibility and relative validity of food group intake in a food frequency questionnaire developed for the Tehran Lipid and Glucose Study. J Epidemiol. 2010;20(2):150-8.

33. Asghari G, Rezazadeh A, Hosseini-Esfahani F, Mehrabi Y, Mirmiran P, Azizi F. Reliability, comparative validity and stability of dietary patterns derived from an FFQ in the Tehran Lipid and Glucose Study. Br J Nut. 2012;108(6):1109-17.

34. Kriska AM, Knowler WC, LaPorte RE, Drash AL, Wing RR, Blair SN, et al. Development of questionnaire to examine relationship of physical activity and diabetes in Pima Indians. Diabetes Care. 1990;13(4):401-11.

35. Momenan AA, Delshad M, Sarbazi N, Rezaei Ghaleh N, Ghanbarian A, Aziz F. Reliability and validity of the Modifiable Activity Questionnaire (MAQ) in an Iranian urban adult population. Arch Iran Med. 2012;15(5):279-82.

36. Azizi F, Khalili D, Aghajani H, Esteghamati A, Hosseinpanah F, Delavari A, et al. Appropriate waist circumference cut-off points among Iranian adults: the first report of the Iranian National Committee of Obesity. Arch Iran Med. 2010;13(3):243-4.

37. MWer S, Dykes D, Polesky H. A simple salting out procedure for extracting DNA from human nucleated cells. Nucleic acids res. 1988;16:1215.

38. Daneshpour MS, Fallah M-S, Sedaghati-Khayat B, Guity K, Khalili D, Hedayati $\mathrm{M}$, et al. Rationale and design of a genetic study on cardiometabolic risk factors: protocol for the Tehran Cardiometabolic Genetic Study (TCGS). JMIR Res Protocols. 2017;6(2):e28.

39. Investigation of gene-diet interactions in the incretin system and risk of type 2 diabetes: the EPIC-InterAct study. Diabetologia. 2016;59(12):2613-21.

40. Corella D, Carrasco P, Sorli JV, Estruch R, Rico-Sanz J, Martinez-Gonzalez $M A$, et al. Mediterranean diet reduces the adverse effect of the TCF7L2rs7903146 polymorphism on cardiovascular risk factors and stroke incidence: a randomized controlled trial in a high-cardiovascular-risk population. Diabetes Care. 2013;36(11):3803-11.

41. Sotos-Prieto M, Smith CE, Lai CQ, Tucker KL, Ordovas JM, Mattei J. Mediterranean diet adherence modulates anthropometric measures by TCF7L2 genotypes among puerto rican adults. J Nutr. 2020;150(1):167-75.

42. Wirström T, Hilding A, Gu HF, Östenson C-G, Björklund A. Consumption of whole grain reduces risk of deteriorating glucose tolerance, including progression to prediabetes. Am J Clin Nutr. 2013;97(1):179-87. 
43. Grant SF. The TCF7L2 Locus: a genetic window into the pathogenesis of type 1 and type 2 diabetes. Diabetes Care. 2019;42(9):1624-9.

44. Wang J, Zhang J, Li L, Wang Y, Wang Q, Zhai Y, et al. Association of rs 12255372 in the TCF7L2 gene with type 2 diabetes mellitus: a metaanalysis. Braz J Med Biol Res. 2013;46(4):382-93.

45. Cauchi S, Choquet H, Gutiérrez-Aguilar R, Capel F, Grau K, Proença C, et al. Effects of TCF7L2 polymorphisms on obesity in European populations. Obesity (Silver Spring). 2008;16(2):476-82.

46. Haupt A, Thamer C, Heni M, Ketterer C, Machann J, Schick F, et al. Gene variants of TCF7L2 influence weight loss and body composition during lifestyle intervention in a population at risk for type 2 diabetes. Diabetes. 2010;59(3):747-50.

47. Mattei J, Qi Q, Hu FB, Sacks FM, Qi L. TCF7L2 genetic variants modulate the effect of dietary fat intake on changes in body composition during a weight-loss intervention. Am J Clin Nutr. 2012;96(5):1129-36.

48. López-Ortiz MM, Garay-Sevilla ME, Tejero ME, Perez-Luque EL. Analysis of the interaction between transcription factor 7 -like 2 genetic variants with nopal and wholegrain fibre intake: effects on anthropometric andmetabolic characteristics in type 2 diabetes patients. Br J Nutr. 2016;116(6):969-78.

49. Bodhini D, Gaal S, Shatwan I, Ramya K, Ellahi B, Surendran S, et al. Interaction between TCF7L2 polymorphism and dietary fat intake on high density lipoprotein cholesterol. PLoS ONE. 2017;12(11):e0188382.

50. McCarthy Ml, Groop PH, Hansen T. Making the right associations. Diabetologia. 2005;48(7):1241-3.

51. Willett W. Nutritional epidemiology. Oxford: Oxford University Press; 2012.

\section{Publisher's Note}

Springer Nature remains neutral with regard to jurisdictional claims in published maps and institutional affiliations.
Ready to submit your research? Choose BMC and benefit from:

- fast, convenient online submission

- thorough peer review by experienced researchers in your field

- rapid publication on acceptance

- support for research data, including large and complex data types

- gold Open Access which fosters wider collaboration and increased citations

- maximum visibility for your research: over 100M website views per year

At BMC, research is always in progress.

Learn more biomedcentral.com/submissions 